\title{
Gas Gangrene of Different Origin Associated with Clostridium perfringens Type A in Three Patients Simultaneously Hospitalized in a Single Department of Orthopedics and Traumatology in Poland
}

\author{
MONIKA BRZYCHCZY-WŁOCH ${ }^{1 \star}$, DOROTA OCHOŃSKA², ANNA PIOTROWSKA ${ }^{2}$ \\ and MAŁGORZATA BULANDA ${ }^{2}$ \\ ${ }^{1}$ Department of Bacteriology, Microbial Ecology and Parasitology, Chair of Microbiology, \\ Jagiellonian University Medical College, Kraków \\ ${ }^{2}$ Department of Infection Epidemiology, Chair of Microbiology, \\ Jagiellonian University Medical College, Kraków
}

Submitted 26 November 2015, accepted 25 March 2016

Abstract

The objective of the study was to perform a comparative analysis of phenotypic and genetic similarity, determination of resistance profiles, detection of toxin-encoding genes and molecular typing of Clostridium perfringens isolates originating from patients with gas gangrene. The study encompassed three patients with a clinical and microbiological diagnosis of gas gangrene who were hospitalized in one of the hospitals of the Kujawsko-Pomorskie province in the same period of time between $8^{\text {th }}$ April 2015 and $20^{\text {th }}$ April 2015. The three C. perfringens isolates studied had identical biochemical profiles. Two isolates had identical resistance patterns, while the third presented a different profile. Using the multiplex PCR method, all isolates showed the presence of cpa gene encoding $\alpha$-toxin; furthermore, the presence of the $c p b 2$ gene encoding $\beta 2$-toxin was confirmed in two isolates. Genotyping with the use of pulsed field gel electrophoresis (PFGE) indicated that the isolates originating from the three studied patients represent three genetically different restrictive patterns which corresponded to three different clones - clone A, clone B and clone C. As a result of the study, it is possible to conclude that the studied patients simultaneously hospitalized in a single Department of Orthopedics and Traumatology developed three different endogenous infections.

Ke y w ords: Clostridium perfringens, epidemiological investigation, gas gangrene, molecular study

\section{Introduction}

Gas gangrene is also known as clostridial myonecrosis (lat. gangrenea gaseosa), a bacterial infection caused by anaerobic Gram-positive bacilli of the genus Clostridium of a severe clinical course (Sołtys-Bolibrzuch, 2012). Clostridium perfringens species is the most commonly isolated etiological factor for gas gangrene (up to 90\% of cases) (Knap, 2004; Stasiak et al., 2007).

The most common symptoms of gas gangrene are sudden, very severe pain, which responds poorly to painkillers, edema and erythema of the skin and soft tissues, epidermoid cysts filled with blood and serum or sanies, or similar fluid discharges from the wound, crepitus to the touch and a characteristic fetid smell. Furthermore, toxemia-related general symptoms present themselves; also, there are manifestations of the toxic shock syndrome and the multiple organ dysfunction syndrome (MODS) (Sussmann et al., 1998; Martirosian, 2007).
A range of extracellular toxins determines C.perfringens pathogenesis. They were assigned consecutive letters of the Greek alphabet. Alpha-toxin ( $\alpha$ ), which is a major toxin, as well as theta-toxin $(\theta)$ and kappatoxin $(\kappa)$ cause the local and systemic symptoms characteristic of gas gangrene (Stevens and Bryant, 2002).

Among healthy individuals, primarily in elderly patients, the percentage of $C$. perfringens carriage ranges from $6 \%$ to $31 \%$ (Sobel et al., 2005). The frequency of gas gangrene occurrence, according to the literature in English, is estimated to be from 0.1 to 1.0 incidences per one million residents a year and is primarily associated with traumas (Sussmann et al., 1998). In the end of the $20^{\text {th }}$ century, in Great Britain, there were $70-80$ cases reported a year; compared with 200-400 in the USA, while Polish estimates mention around 100 cases of gas gangrene a year (Knap, 2004).

Due to its high toxicity, which results in invasiveness, the species $C$. perfringens can be considered alert microorganisms in accordance with the regulation of

\footnotetext{
* Corresponding author: M. Brzychczy-Włoch, Department of Bacteriology, Microbial Ecology and Parasitology, Jagiellonian University Medical College, Krakow, Poland; e-mail: mbrzych@cm-uj.krakow.pl
} 
the Minister of Health in Poland of $23^{\text {rd }}$ December 2011 concerning the list of alert factors, hospital infections and alert factors records as well as reports on the current epidemiological situation of the hospital (Ministerial Decree, 2011).

Microbiological diagnostic analysis, together with clinical picture, form the basis for diagnosing gas gangrene. The former requires proper sampling of the material for testing, which is either a tissue fragment or sanies from the border of healthy tissues, and when it is impossible to obtain biopsy material, a swab is taken. Biological material makes it possible to prepare a direct smear which should be stained using the Gram stain technique and tested under the microscope for Grampositive cylindrical forms. It is common for direct smears not to contain leucocytes. It results from lytic activity of lecithinase and theta-toxin. Simultaneously, a culture for aerobic and anaerobic bacteria should be prepared using the biological material, which enables a conclusive confirmation of C. perfringens occurrence. Tissue biopsy material taken from the site affected by gas gangrene is subjected to a histopathological test to enable, in a way similar to direct smear, quick preliminary confirmation of the diagnosis. In order to diagnose gas gangrene and implement treatment, it is enough to have a clinical picture of the patient and detect the presence of Gram-positive cylindrical forms in the direct smear, while the C. perfringens culture serves to confirm the diagnosis (Sussmann et al., 1998; Martirosian, 2007).

Molecular methods employed for bacterial isolates typing make it possible to determine the genetic similarity of species originating either from infections or from hospital environment (van Belkum et al., 2007).

For C. perfringens the toxin typing is very important because of particular toxin types are associated with the specific clinical course of the disease (Stevens and Bryant, 2002). A multiplex PCR method simultaneously detecting the four major toxin genes $(c p a, c p b$, etx, iap), the enterotoxin gene (cpe), and all variants of the beta2 toxin gene (cpb2) (van Asten et al., 2009).

Pulsed field gel electrophoresis (PFGE), which is recognized as the gold standard in microbial typing, make it possible to compare the genetic similarity of C. perfringens isolates, which permits genetically-identical bacterial clones to be selected (van Belkum et al., 2007). It is a very helpful tool applied in epidemiological investigation of gas gangrene cases reported in patients hospitalized in the same hospital unit (Maslanka et al., 1999; Brzychczy-Włoch et al., 2014).

Objective of the study. The objective of the study was to perform a comparative analysis of phenotypic and genetic similarity of C.perfringens isolates originating from three patients with gas gangrene simultaneously hospitalized in a single Department of Orthopedics and Traumatology in 2015.

\section{Experimental}

Materials and Methods

The study encompassed three patients with a clinical and microbiological diagnosis of gas gangrene who were hospitalized in one of the hospitals of the Kujawsko-Pomorskie province in the time period between $15^{\text {th }}$ April 2015 and $20^{\text {th }}$ April 2015.

\section{Description of the cases}

Patient 1. A man aged 53 was admitted to the Department of Orthopedics and Traumatology on 23 March 2015 as emergency inpatient with a fractured left tibial epiphysis due to a fall from a ladder on the day of the admission. On 24 March a plaster splint was applied and traction was used. Due to lower leg swelling, a surgical procedure was postponed. From 23 March to 3 May, the patient received amoxicillin with clavulanic acid $3 \times 1.2 \mathrm{~g}$ IV. On 2 April, the surgical procedure was performed. Perioperative prophylaxis was provided with cefazolin $2 \mathrm{~g}$ IV. After 8 days on 10 April, the patient developed a fever. A knee puncture was performed. Bloody fluid was taken from the knee and sent for testing. Enterococcus faecalis was cultured aerobically and C.perfringens was cultured anaerobically from the material. The targeted therapy was used. Clindamycin was administered $3 \times 600 \mathrm{mg}$ IV. On 15 April, wound debridement was carried out and intraoperative material was taken. C. perfringens was cultured anaerobically from the material (lab no. 7013); aerobically, there was no growth. On 23 April, clindamycin was discontinued and metronidazole was started at a dose of $0.5 \mathrm{~g} \mathrm{IV} \times 8 \mathrm{~h}$ until 3 May. Wound irrigation was performed using hydrogen peroxide. Patient isolation was implemented. The material from the patient was collected several times more until 2 May - cultures were negative. Consultation with Hyperbaric Oxygen Center in Gdansk was done. The patient was qualified for treatment as the wound was healing slowly, but the treatment was not applied. April, 24: wound debridement, removal of necrotic sections. Aquacel Ag (ConvaTec Poland) foam dressing. April, 28: VivanoMed (Hartmann) vacuum dressing was applied for about 3 weeks. On 2 June, after 102 days of hospitalization the patient was discharged from hospital in a good condition.

Patient 2. A woman aged 88 was admitted to the Department of Orthopedics and Traumatology on 26 March 2015 after a fall at home. The diagnosis: a closed comminuted fracture of the right femur. Comorbidities: type 2 diabetes, atherosclerotic cardiomyopathy, circulatory failure NYHA III, hypertension, cholecystolithiasis, erosive gastritis. On the day of admission, 3 units of packed red blood cells (PRBCs) 
were transfused. On 27 March, a surgical procedure was carried out involving fracture fixation with a stainless-steel popliteal intramedullary nail (Megdal). Perioperative prophylaxis was used - cefazolin $2 \mathrm{~g}$ IV. The patient was agitated on 28 April during the night, periodically without contact. April, 30: patient's condition is stable. On 2 April, moderately severe condition. Due to destabilization, reoperation to remove the fixation and an NCB (Zimmer) titanium femur plate was placed. After $24 \mathrm{~h}$, the swelling without symptoms of crepitus of the affected limb. Two units of PRBCs were transfused and material from the wound was collected. On 7 April, ciprofloxacin was administered $2 \times 750 \mathrm{mg}$ IV until 13 April. On 9 April, material was taken for testing. There was lack of growth of aerobic flora (there was no culture for anaerobic bacteria). Lack of progress in wound healing. Discharge of a small amount of serum. Octenisept (Schulke S\&M) rinsing was employed and Cosmopor (Hartmann) dressings. On 17 April, material for testing was collected. C. perfringens (lab no. 7149) was cultured; aerobically, no growth. From 17 April, amoxicillin with clavulanic acid was started $3 \times 2.4 \mathrm{~g}$ IV in combination with clindamycin $3 \times 600 \mathrm{mg}$ IV until 20 April. On the night of 19 April, deterioration of patient's condition. Symptoms of circulatory failure. The patient received oxygen therapy. April, 20, on the $26^{\text {th }}$ hospital day: Patient's death due to multiorgan failure.

Patient 3. A woman aged 76 was admitted to the Department of Orthopedics and Traumatology on 8 April 2015 as emergency inpatient. As a result of a fall at home, she suffered a right thigh injury. Diagnosis: comminuted closed fracture of the femur. Comorbidities: hypertension, ischemic heart disease, type 2 diabetes. On 9 April, supramalleolar traction was employed. On 14 April, MIPO (minimally invasive plate osteosynthesis) thigh fixation surgery was carried out with the use of the NCB (Zimmer) titanium plate. April, 17: deterioration of the patient's condition, temperature increase to $38.9^{\circ} \mathrm{C}$, pain in the limb, skin bruising. During the exam, a subcutaneous space was found, which showed symptoms of crepitus upon palpitation. The necrotic skin was incised with evidence of gas release and discharge of a significant amount of turbid dark liquid. Material for microbiological testing was collected. C.perfringens (lab no. 7143) was cultured; aerobically - no growth. The resulting fistula was rinsed with hydrogen peroxide. From 17 April, amoxicillin with clavulanic acid was prescribed $3 \times 1.2 \mathrm{~g}$ in combination with clindamycin $3 \times 600 \mathrm{mg}$ IV. In view of the fulminant gas gangrene, on 17 April, a decision was made to amputate the limb, however, the patient did not consent to surgery. On 18 April, after obtaining the patient's consent to amputate the limb, the life-saving procedure was undertaken.
During the surgery, CPR was performed several times on the patient. Death occurred on the $10^{\text {th }}$ hospital day during surgery. Final diagnosis: death due to infection with C. perfringens.

Microbiological diagnostics. Materials for microbiological examination from the three studied patients were taken using sterile swabs for the Amies (COPAN) activated carbon substrate. Culturing was carried out on the Columbia Sheep Blood Agar (BioMérieux) medium under aerobic and anaerobic conditions as well as on liquid thioglycollate medium with resazurin (BioMaxima). Solid media with the inoculated material were incubated under aerobic conditions at $36^{\circ} \mathrm{C} / 48 \mathrm{~h}$ and under anaerobic conditions at $36^{\circ} \mathrm{C} / 24 \mathrm{~h}$ and the liquid medium under aerobic conditions (with a closed cap) at $36^{\circ} \mathrm{C} / 48 \mathrm{~h}$. Identification of the cultured bacteria was carried out using the Vitek 2 compact system (BioMérieux). The biochemical patterns for C.perfringens isolates were received with the use of API 20A test (BioMérieux).

The following strains of C.perfringens were protected for the further studies: from patient 1 - isolate no 7013; from patient 2 - isolate no 7149; from patient 3 - isolate no 7143. C. perfringens 12915 ATCC (The American Type Culture Collection) standard was used as reference strain. The strains were stored with the use of Cryobank (BioMaxima) at $-70^{\circ} \mathrm{C}$.

Protection of epidemic outbreak and epidemiological investigation. On 15 April 2015, the ward and the epidemiological nurse were informed by phone by the Department of Microbiology Diagnostics about the isolation of an alert pathogen. The nurse went to the unit to arrange a method for patient isolation. Since the patient was in the Main Operating Theater, the Octenisept (Schulke S\&M) sporicidal agent was used (for debridement and rinsing of the wound).

On 17 April, a meeting was held of the Hospital Infection Control Team and the Head of the Department of Orthopedics and Traumatology on account of culturing an alert pathogen in patient no. 1 (lab no. 7013) and a suspicion in patient no. 3 (lab no. 7143 ). Intensification of the sanitary regime was established, together with isolation of patient no. 3, application of the Incidin Active (Ecolab) sporicidal agent for surface decontamination. Aniosept activ (Medilab) was used for surfaces and tools in the Main Operating Theater. Equipment was allocated to the isolated patients. An inspection was carried out of the compliance with handling medical waste and bed linen (labeling of notifications according to the procedure in double red bags). The Head of the Main Operating Theater was instructed to execute preventative decontamination of all operating rooms with a sporicidal agent and to support it by fogging the rooms with HyPro technical 6-7\% $\mathrm{H}_{2} \mathrm{O}_{2}$ using the Hyspray (Hymetec) appliance. 
On 18 April, after oral information from the Department of Microbiological Diagnostics concerning the suspicion of $C$. perfringens culture in patient no. 2 (lab no. 7149), patient isolation was continued, sanitary regime was intensified, and separate staff was assigned to the isolated patients.

On 19 April, all the patients of the Ward were included into the outbreak - a total of 20 people. Action taken: contact isolation of patients; intensification of sanitary regime; dedication of separate equipment to isolated patients; assignment of staff to care for isolated patients; environmental control after each decontamination procedure.

On 20 April, the Director of the Hospital decided not to perform the scheduled surgical procedures and to execute another general disinfection of the operating rooms and equipment in conjunction with fogging and taking environmental tests, including from the hands of workers in the operating theater, employees and surgical wards.

On 21 April until 29 April, in accordance with the decision of the District Sanitary Inspector, planned admissions to the surgical wards and scheduled surgical procedures were suspended. At that time, decontamination of rooms was carried out together with fogging and all surgical instruments were subjected to reprocessing.

Between 19 and 24 April, daily meetings were held involving the Infection Control Committee and the Hospital Administration with the purpose of discussing the current situation.

Inspection of the decontamination of surfaces and hygienic hand washing was carried out by the contact plate method using Count-Tact (BioMérieux) system with the use of Agar Count-Tact (BioMérieux) radiation-sterilized plates. Incubation of the plates under aerobic conditions at $30^{\circ} \mathrm{C} / 72 \mathrm{~h}$.

After the exhaustion of the stock of plates, further examination was carried out using swabs. Materials were collected on a sterile swab (COPAN) and subsequently placed in Tryptic Soy Broth (BioMaxima). Incubation was carried out at $36^{\circ} \mathrm{C} / 72 \mathrm{~h}$. Positive liquid cultures were plated on solid media: Columbia Agar, MacConkey Agar and Sabouraud Agar (BioMérieux). Each material was tested for aerobic and anaerobic flora.

Antibiotic susceptibility testing. To determine the drug-resistance profiles, the Kirby-Bauer disk diffusion method was used in which the Columbia Sheep Blood Agar (BioMérieux) and antibiotic disks (Oxoid) were utilized: penicillin - P [1 U], imipenem - IPM $[10 \mu \mathrm{g}]$, amikacin - AK [30 $\mu \mathrm{g}]$, gentamicin - CN $[10 \mu \mathrm{g}]$, tobramycin - TOB $[10 \mu \mathrm{g}]$, netilmicin - NET [10 $\mu \mathrm{g}$ ], metronidazole - MTZ [5 $\mu \mathrm{g}$ ], chloramphenicol - C [30 $\mu \mathrm{g}]$ and the E-test method enabling determination of MIC (Minimal Inhibitory Concentration) for: ampicillin - AM, clindamycin - DA, doripenem
- DOR, doxycycline - DC, ciprofloxacin - CI, vancomycin - VA (bioMerieux). The results were interpreted according to The European Committee on Antimicrobial Susceptibility Testing (EUCAST) version 5.0 (EUCAST, 2015).

PCR multiplex. To isolate DNA, the Genomic Mini Set (A\&A Biotechnology) was used according to the manufacturer's protocol. The presence of genes encoding toxins of $C$. perfringens was confirmed using multiplex PCR amplification according to van Asten etal. (van Asten etal., 2009) with specific primers (Genomed). The following genes were detected: $c p a$ 324 bp ( $\alpha$-toxin); cpb 195 bp ( $\beta$-toxin); cpb2 548 bp ( $\beta 2$-toxin); etx 376 bp ( $\varepsilon$-toxin); iap 272 bp ( $\zeta$-toxin); cpe 485 bp (enterotoxin). The final pictures from electrophoresis were processed using QuantityOne software, as well as GelDoc2000 device (Bio-Rad, USA).

Molecular typing with PFGE. The chosen C.perfringens isolates underwent molecular typing using the PFGE method according to the methodology described by Maslanka et al. (1999). Chromosomal DNA of bacterial strains was isolated in agarose blocks and then digested with the use of restriction enzyme Smal (MBI Fermentas). Electrophoretic separation was performed on CHEF-DR II (Bio-Rad) machine, while restriction analysis was carried out using GelCompar II (Applied Maths) software with the application of UPGMA clustering method and Jaccard index. The obtained genetic profiles were interpreted according to the guidelines given by van Belkum et al. (2007).

\section{Results}

The diagnosis of gas gangrene made on the basis of clinical picture in the three patients hospitalized in the Department of Orthopedics and Traumatology of the district hospital was confirmed with microbiological testing, in which C.perfringens strains were isolated from the intraoperative clinical materials: patient 1 - isolate no. 7013; patient 2 - isolate no. 7149; patient 3 - isolate no. 7143 .

The first strain of C.perfringens no. 7013 was isolated on 10 April 2015 (patient 1), and reported to the ward and the epidemiological nurse on 15 April. The second and third strains were isolated on 17 April (patient 2 and 3). On 6 May 2015, activities associated with eradicating the outbreak were completed.

During the epidemiological investigation, materials were taken in the amount of 173 from patients of the Department of Orthopedics and 140 from the staff of the Department of Orthopedics, General Surgery, Anaesthesia and Intensive Care, and the Main Operating Theater. 250 materials were also taken from the hospital environment (mainly the ward and operating 
Table I

Biochemical profiles obtained with the using of the API 20A test (BioMérieux).

\begin{tabular}{|c|c|c|c|c|c|c|c|c|c|c|c|c|c|c|c|c|c|c|c|c|c|c|c|c|}
\hline $\begin{array}{l}\text { Patient / } \\
\text { Isolate no. }\end{array}$ & \multicolumn{24}{|c|}{ Biochemical tests } \\
\hline Patient 17013 & - & - & + & - & + & + & + & - & - & - & + & - & - & - & + & - & - & - & - & + & - & + & + & - \\
\hline Patient 27149 & - & - & + & - & + & + & + & - & - & - & + & - & + & - & + & - & - & - & - & + & - & + & + & - \\
\hline
\end{tabular}

“+”- positive reaction; “-” - negative reaction

theater). None of the materials enabled culturing the C. perfringens alert pathogen.

With the use of API 20A (BioMérieux) test, biochemical profiles of the isolates tested were established (Table I) and they were identical for the isolates no. 7149 and no. 7143 (the second and the third patient) and different for isolate no. 7013 (the first patient).

Table II

Antibiotic susceptibility for C. perfringens isolates.

\begin{tabular}{|l|c|c|c|}
\hline & $\begin{array}{c}\text { Patient 1/ } \\
\text { Isolate } \\
\text { no 7013 }\end{array}$ & $\begin{array}{c}\text { Patient 2/ } \\
\text { Isolate } \\
\text { no 7149 }\end{array}$ & $\begin{array}{c}\text { Patient 3/ } \\
\text { Isolate } \\
\text { no 7143 }\end{array}$ \\
\hline $\begin{array}{c}\text { The Kirby-Bauer } \\
\text { disk diffusion method }\end{array}$ & \multicolumn{3}{|c|}{ Inhibition zone [mm] } \\
\hline Penicillin & 35 & 35 & 35 \\
\hline Imipenem & 35 & 35 & 35 \\
\hline Amikacin & 32 & 24 & 5 \\
\hline Gentamicin & 30 & 22 & 5 \\
\hline Tobramycin & 30 & 22 & 5 \\
\hline Netilmicin & 30 & 22 & 5 \\
\hline Metronidazole & 5 & 5 & 5 \\
\hline Chloramphenicol & 35 & 35 & 35 \\
\hline \multicolumn{1}{|c|}{ The E-test method } & Minimal inhibitory concentration; \\
\hline Ampicillin & 0.016 & 0.016 & 0.016 \\
\hline Clindamycin & 0.064 & 0.19 & 48 \\
\hline Doripenem & 0.064 & 0.064 & 0.064 \\
\hline Doxycycline & 0.19 & 0.125 & 2 \\
\hline Chloramphenicol & 0.25 & 0.19 & 0.25 \\
\hline Vancomycin & 0.016 & 0.016 & 0.016 \\
\hline
\end{tabular}

Using the Kirby-Bauer method and E-test, drugresistance patterns of the studied isolates were determined (Table II). In the EUCAST version 5.0 for 2015 the criteria for determining susceptibility testing of anaerobic bacteria by disc diffusion method not yet been determined. However, in the case of the first and second patient, the isolated strains no. 7013 and 7149 had the same drug-resistance patterns, while the isolate no. 7143 from the third patient had different antibiotic drug resistance profile. Using the E-test method the susceptibility to ampicillin, clindamycin, doripenem, doxycycline, ciprofloxacin and vancomycin were detected for the study isolates. Only isolate no. 7143 from the third patient was resistant to clindamycin.

When multiplex PCR was applied, all C.perfringens isolates originating from the three patients demonstrated the presence of cpa gene encoding a-toxin. Moreover, in isolate no. 7149 coming from patient 2 and in isolate no. 7143 from patient 3, cpb2 gene encoding $\beta 2$-toxin presence was confirmed (Fig. 1; Table III).

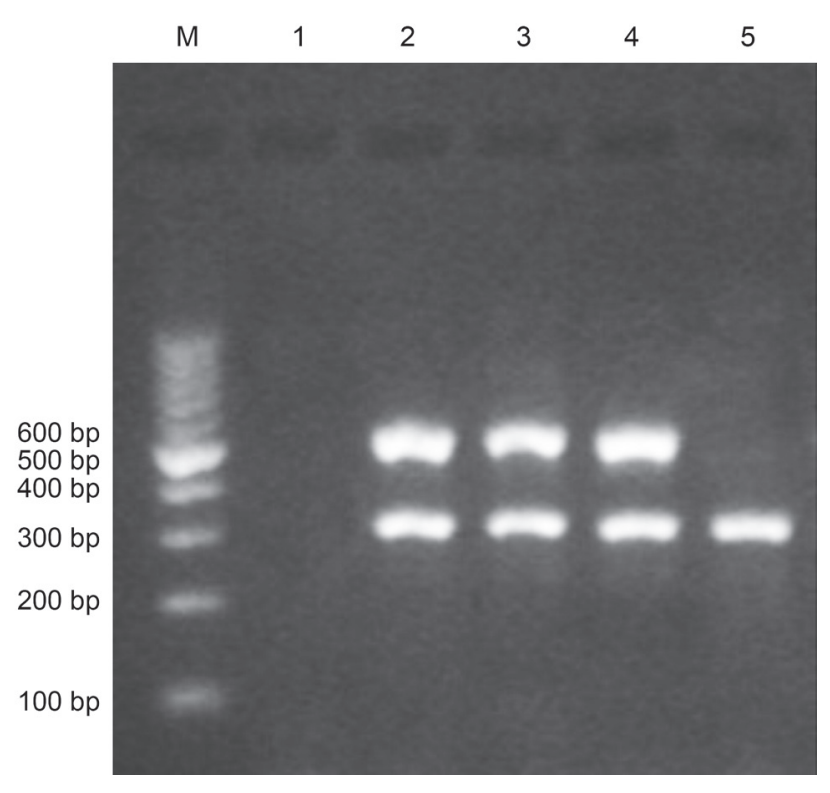

Fig. 1. Detection of genes encoding virulence factors of C. perfringens by multiplex PCR method.

$\mathrm{M}$ - size marker; 1 - negative control $\left(\mathrm{H}_{2} \mathrm{O}\right) ; 2$ - reference strain of C. perfringens ATCC 12915; 3 - C. perfringens isolate no. 7143 from patient 3; 4 - C. perfringens isolate no. 7149 from patient 2; 5 - C. perfringens isolate no. 7013 from patient 1.

C. perfringens isolates genotyping with the use of pulsed field gel electrophoresis (PFGE) indicated that the isolates originating from the three studied patients represent three genetically different restriction patterns and are related to each other in $66.7 \%$ (isolates no. 7143 and 7149 ) and in $46.4 \%$ (isolates no. 7143,7149 and 7013). The isolates studied represented three different 
Table III

Occurrence of genes encoding virulence factors in C. perfringens isolates.

\begin{tabular}{|l|c|c|c|c|c|}
\hline Gene & $\begin{array}{c}\text { Size of the ampli- } \\
\text { fication product }\end{array}$ & Toxin & $\begin{array}{c}\text { Patient 1/ } \\
\text { Isolate no. 7013 }\end{array}$ & $\begin{array}{c}\text { Patient 2/ } \\
\text { Isolate no. 7149 }\end{array}$ & $\begin{array}{c}\text { Patient 3/ } \\
\text { Isolate no. 7143 }\end{array}$ \\
\hline$c p a$ & $324 \mathrm{bp}$ & a-toxin & $c p a$ & $c p a$ & $c p a$ \\
\hline$c p b$ & $195 \mathrm{bp}$ & $\beta$-toxin & - & - & - \\
\hline$c p b 2$ & $548 \mathrm{bp}$ & $\beta 2$-toxin & - & $c p b 2$ & $c p b 2$ \\
\hline etx & $376 \mathrm{bp}$ & $\varepsilon$-toxin & - & - & - \\
\hline$i a p$ & $272 \mathrm{bp}$ & t-toxin & - & - & - \\
\hline$c p e$ & $485 \mathrm{bp}$ & enterotoxin & - & - & - \\
\hline
\end{tabular}

PFGE types, which corresponded to three different clones - clone A (isolate no. 7143), clone B (isolate no. 7149) and clone C (isolate no. 7013) (Fig. 2). gens etiology, 15-20\% of isolates had this toxin (Fisher et al., 2005). Probably the synergic interactions of both toxins is of great importance.
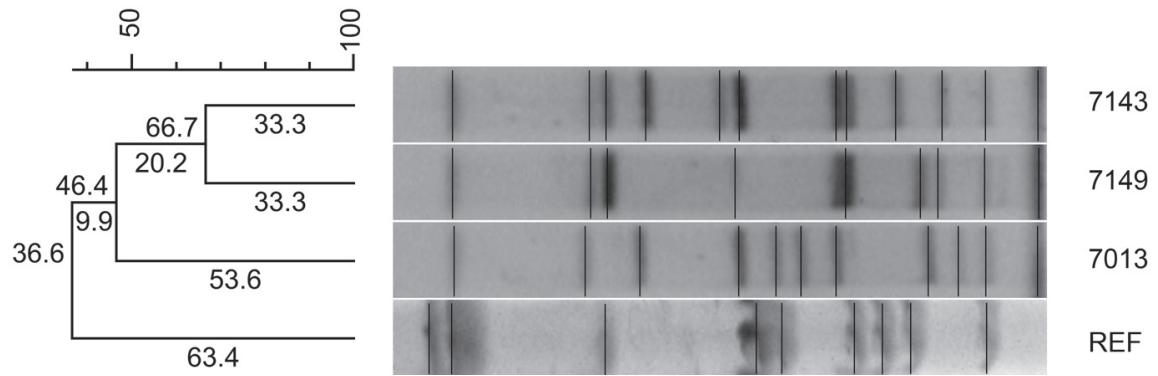

Fig. 2. Analysis of genetic profiles of C. perfringens isolates, subjected to DNA digestion by restriction enzyme SmaI, carried out with the use of pulsed field gel electrophoresis (PFGE) method and GelCompare II software. Legend: 7143 - C. perfringens isolate from patient 3; 7149 - C. perfringens isolate from patient 2; 7013 - C. perfringens isolate from patient 1; REF - C. perfringens reference strain ATCC 12915.

\section{Discussion}

Nowadays, in the USA about 1000 cases of gas gangrene are recorded each year (Abella et al., 2003). In Poland there are no epidemiological data concerning this issue.

The three clinical cases of gas gangrene associated with C.perfringens described in this paper, ending with the death of two patients, confirm the fact that gas gangrene is an extremely severe surgical infection associated with high mortality. In our study the mortality rate was $66,7 \%$. Presently, gas gangrene is very rare, however, similar cases were described in Polish literature in the past (Wojdat et al., 2005; Stasiak et al., 2007; Brzychczy-Włoch et al., 2014).

The most important virulence factor of C.perfringens is a-toxin encoded by the gene cpa which possesses activities of lecithinase and haemolysine. $\alpha$-toxin causes damage and lysis of cell membrane which has a lethal effect to different tissues. Moreover, this toxin has dermonecrotic properties and inhibits functions of neutrophils (Stevens and Bryant, 2002). Gibert et al. (1997) described the new toxin with cytotoxic ability called the $\beta 2$-toxin (CPB2) encoded by a gene $c p b 2$. In a study on patients with food poisoning of C.perfrin-
With the use of multiplex PCR we detected the presence of cpa gene encoding a-toxin in all C. perfringens isolates originating from the three patients. Moreover, in two isolates from patient 2 and 3 the $c p b 2$ gene encoding $\beta 2$-toxin presence was confirmed. Described in the literature cases of gas gangrene were associated with the presence of $a$-toxin (Stevens and Bryant, 2002; Stasiak et al., 2007). It is worth noting that in our study the presence of strains with additional $\beta 2$-toxin, was associated with severe course of infection and the death of two patients.

Due to the fact that gas gangrene progresses rapidly and combined with irreversible soft tissue changes, the success of therapy very much depends on the time of the diagnosis on the basis of the clinical picture, bacteriological study results and the implementation of treatment. The close cooperation between a microbiological laboratory and a surgical staff is essential in proper management of gas gangrene (Sussmann et al., 1998; Martirosian, 2007).

Crystalline penicillin applied intravenously in high doses (e.g. 24 units per $24 \mathrm{~h}$ ), most often combined with metronidazole, is the most often selected antibiotic in the treatment of gas gangrene. Supplementary effective antibiotics are aminoglycosides, lincosamides e.g. 
clindamycin, semisythetic penicillins e.g. amoxicillin, ticarcillin combined with bacterial $\beta$-lactamase inhibitors e.g. clavulanic acid, fluoroquinolones and tetracyclines coupled with aminoglycosides (Knap, 2004; Martirosian, 2007).

In three patients described, after isolation of C. perfringens from clinical materials, amoxicillin with clavulanic acid in combination with clindamycin were used. However, despite adequate treatment implemented two of the patients died due to infection with C.perfringens and multiple organ failure.

In order to limit the epidemic outbreak a number of measures to identify the outbreak and to limit the spread of infection were taken. Unfortunately, in the cases described highlights a very long time (5 days) since the first strain of C.perfringens from the first patient were isolated, to forward this information to the Department of Orthopedics and the Hospital Infection Control Team. In the circumstances lacked a rapid response to the isolation of alert pathogen, which was associated with a high risk to other patients and the possibility of spread of the epidemic outbreak to other departments.

Microbiological studies were carried out in patients of the Department of Orthopedics, in the staff of the Department of Orthopedics, General Surgery, Anaesthesia and Intensive Care, and the Main Operating Theater and in the hospital environment. Despite the huge number of materials testing (total 563) in any case C.perfringens was not isolated. It indicates that the pathogen was not spread to other patients and did not get to the hospital environment.

The treatment of gas gangrene should be complex. It consists in debridement, employment of empirical and targeted antibiotherapy as well as hyperbaric therapy. Hyperbaric oxygen therapy (HBOT) is effective in limiting the number of toxins produced by $C$. perfringens that cause tissue necrosis in gas gangrene, and coupled with antibiotherapy, it reduces the time needed for treatment and significantly lowers mortality (Sussmann et al., 1998; Fielden et al., 2002; Martirosian, 2007). In the first patients described efforts were made to implement the HBOT but unfortunately it was not applied. Implementation of HBOT certainly would shorten the time of his hospitalization, which was as high as 102 days, after which the patient in good condition was discharged at home.

Antiseptics effective against $C$. perfringens including spores are: $0.1 \%$ Octenisept, $10 \%$ Betadine or $5 \%$ Hibitane (Stasiak et al., 2007). In described cases Octenisept rinsing was employed for debridement and rinsing of the wound.

As shown in the studies, phenotypic characteristics, including the determination of biochemical profiles and patterns of antibiotic resistance is inadequate in differentiating strains of C.perfringens. Molecular typing is of paramount importance in epidemiological investigation testing, which tries to detect causes, sources and mechanisms of microorganisms spreading among patients, e.g. in a single hospital units (Fleischer and Salik, 2006; Brzychczy-Włoch et al., 2014). Detailed molecular studies using the PFGE method showed that C. perfringens strains isolated from the three patients represented three different genetic clones. This demonstrates the different origins of these infections, which was the most likely source of endogenous flora in these patients. Similar results were also described earlier by our team, where there was an infection of $C$. perfringens of various origins in two patients simultaneously hospitalized in a single department (Brzychczy-Włoch et al., 2014). Similar results were also described by Stasiak et al. (2007), who presented two patients with severe gas gangrene syndromes of a completely different origin.

\section{Conclusions}

As a result of the epidemiological and molecular study, it is possible to conclude that the three studied patients simultaneously hospitalized in a single Department of Orthopedics and Traumatology with diagnosed gas gangrene developed three different endogenous infection.

\section{Acknowledgements}

The authors would like to thank the employees of the Central Laboratory of the district hospital for their cooperation and assistance in conducting the study. The authors wish to acknowledge also to PhD Tomasz Gosiewski and M.Sc. Anna Malska-Woźniak for technical assistance in the PFGE method.

Publication was supported by the Faculty of Medicine of Jagiellonian University Medical College (Leading National Research Centre 2012-2017).

\section{Literature}

Abella B.S., P. Kuchinic, T. Hiraoka and D.S. Howes. 2003. Atraumatic Clostridial myonecrosis: case report and literature review. J. Emerg. Med. 24(4): 401-405.

Brzychczy-Włoch M. and M. Bulanda. 2014. Analysis of genetic similarities between Clostridium perfringens isolates isolated from patients with gas gangrene and from hospital environment conducted with the use of the PFGE method. Pol. J. Surg. 86(3): 141-146.

Fielden M.P., E. Martinovic and A.L. Ells. 2002. Hyperbaric oxygen therapy in the treatment of orbital gas gangrene. JAAPOS. 6 : 252-254.

Fisher D.J, K. Miyamoto, B. Harrison, S. Akimoto, M.R. Sarker and B.A. McClane. 2005. Association of beta2 toxin production with Clostridium perfringens type A human gastrointestinal disease isolates carrying a plasmid enterotoxin gene. Mol. Microbiol. 56(3): 747-762. 
Fleischer M. and K. Salik. 2006. Procedures in the hospital outbreaks. Training materials for epidemiological nurses (in Polish). Wrocław. http://pspe.pl/files/pliki_old/zaszyt-V.pdf, 2015.11.01. Gilbert M., C. Jolivet-Reynaud and M.R. Popoff. 1997. Beta2 toxin, a novel toxin produced by Clostridium perfringens. Gene 203: 65-73. Knap J. 2004. Gas gangrene, pp. 411-414. In: W. Magdzik, D. Narszewicz-Lesiuk and A.Zieliński (eds.) Infectious and parasitic diseases - epidemiology and prevention (in Polish). $\alpha$-medica Press, Bielsko-Biała.

Martirosian G. 2007. Infections caused by Clostridium perfringens, gas gangrene, food poisoning, pp. 646-649. In: J. Cianciara and J. Juszczyk (eds). Infectious and parasitic diseases (in Polish). Czelej Sp. z o.o., Lublin.

Maslanka S.E., J.G. Kerr, G. Williams, J.M. Barbaree, L.A. Carson, J.M. Miller and B. Swaminathan. 1999. Molecular subtyping of Clostridium perfringens by pulsed-field gel electrophoresis to facilitate food-borne-disease outbreak investigations. J. Clin. Microbiol. 37: 2209-2214.

Regulation of the Minister of Health of 23 December 2011 on the list of factors alarm registers of hospital infections and factors alarms and reports on the current epidemiological situation of the hospital (in Polish). Dz.U.11.294.1741.

Sobel J., C.G. Mixter, P. Kolhe, A. Gupta, J. Guarner, S. Zaki, N.A. Hoffman, J.G. Songer, M. Fremont-Smith, M. Fischer and others. 2005. Necrotizing enterocolitis associated with Clostridium perfringens type A in previously healthy North American adults. J. Am. Coll. Surg. 201: 48-56.
Soltys-Bolibrzuch K. 2012. The new microorganism on the list of alarm factors - Clostridium perfringens. Treatment of the patient in case of suspicion and/or diagnosis of gas gangrene (in Polish). Pielegniarka Epidemiologiczna 1: 5-7.

Stasiak M., J. Lasek, A. Samet, A. Śledzińska, Z. Witkowski, E. Augustynowicz, W. Marks and J. Ślusarczyk. 2007. Gas gangrene of a different origin in trauma patients - report of 2 cases. J. Orthopaedics 4(1): e15.

Stevens D.L. and A.E. Bryant. 2002. The role of clostridial toxins in the pathogenesis of gas gangrene. CID. 35: 593-600.

Sussmann M., S.P. Boriello and D.J. Taylor. 1998. Gas gangrene and other clostridial infections, pp. 669-684. In: Collier L., A. Balows and M. Sussman (eds). Topley and Wilsons' microbiology and microbial infections, $9^{\text {th }} \mathrm{ed}$. Arnold, London.

The European Committee on Antimicrobial Susceptibility Testing (ECAST). 2015. Breakpoint tables for interpretation of MICs and zone diameters. Version 5.0, 2015. http://www.eucast.org, 2015.11.01 van Asten A.J., C.W. van der Wiel, G. Nikolaou, D.J. Houwers and A. Gröne. 2009. A multiplex PCR for toxin typing of Clostridium perfringens isolates. Vet. Microbiol. 136(3-4): 411-412.

van Belkum A., P.T. Tassios, L. Dijkshoorn, S. Haeggman, B. Cookson, N.K. Fry, V. Fussing, J. Green, E. Feil, P. GernerSmidt and others. 2007. Guidelines for the validation and application of typing methods for use in bacterial epidemiology. Clin Microbiol. Infect. 13: 1-46.

Wojdat E., K. Kwiatek, H. Zdrojewski and I. Krupa. 2005. Gas gangrene - case diagnosis (in Polish). Przegl. Epidemiol. 59: 859-863. 